\title{
Análise de sustentabilidade: Índice De Sustentabilidade Empresarial Bovespa e carteiras 2013, 2014 e 2015
}

O tema sustentabilidade nos últimos anos, vem sendo considerado assunto pertinente e presente na sociedade, devido ao impacto que o mesmo resulta no nosso cotidiano, diante do agravamento dos problemas ambientais ocorridos e do uso indiscriminado dos recursos naturais existentes, assiste-se à emergência de um novo tipo de postura dos indivíduos, a qual pondera os impactos de seu padrão de consumo na natureza. Assim sendo, o objetivo do estudo é mostrar a evolução do tema meio ambiente, sustentabilidade e a evolução da Bovespa e parceiros na inserção do tema junto as empresas envolvidas na carteira do Índice de Sustentabilidade Empresarial Bovespa (ISE). O método de pesquisa foi de abordagem quantitativa, com finalidade exploratória e descritiva, por meio de pesquisa documental. Evidenciou-se que as empresas estão construindo seus pilares na área da sustentabilidade, por ser um tema amplo compreende-se que os modelos já existentes servem como norteadores das empresas que possuem o mesmo segmento. A gestão ambiental é um assunto de interesse geral das nações, por essa razão demonstra-se a importância da elaboração de práticas eficazes por parte das empresas, assim como a divulgação delas para que sirvam como fonte de informações na tomada de decisões por parte dos diversos usuários

Palavras-chave: Sustentabilidade; Sustentabilidade Empresarial; Índices.

\section{Sustainability analysis: ISE Bovespa and wallets 2013, 2014 and 2015}

The sustainability issue in recent years has been considered relevant and this subject in society, due to the impact that the same results in our daily lives, before the worsening occurred environmental problems and the indiscriminate use of natural resources, we are witnessing the emergence of a new kind of posture of individuals, which considers the impact of their consumption pattern in nature. Therefore, the objective of the study is to show the evolution of the theme environment, sustainability and evolution of the Bovespa and partners in the theme of integration with the companies involved in the portfolio of the Corporate Sustainability Index (ISE). The research method has qualitative with descriptive purpose, as a technical document search procedure. It was evident that companies are building their pillars in the area of sustainability, as a broad theme is understood that the existing models serve as guiding companies that have the same segment. Environmental management is a matter of general interest of the nation, therefore it demonstrates the importance of developing effective practices by companies, as well as posting them to serve as source of information in making decisions by many users.

Keywords: Sustainability, Corporate Sustainability, Index.

Topic: Valoração e Economia Ambiental

Reviewed anonymously in the process of blind peer.
Received: 06/10/2016

Approved: 12/01/2017
Alessandra dos Santos Welter

rrmussi@yahoo.com.br

\section{Rejane Remussi}

Fundação Universidade de Caxias do Sul, Brasil.

http://lattes.cnpq.br/5611017055859229

rrmussi@yahoo.com.br

Pelayo Munhoz Ólea

Universitat Politècnica de Catalunya, Espanha.

http://lattes.cnpq.br/6209414364209633

pelayo.olea@gmail.com

\section{Alice Munz Fernandes}

Universidade Federal do Rio Grande do Sul, Brasil.

http://lattes.cnpq.br/4179890354485915

alicemunz@gmail.com

\author{
Uiliam Hahn Biegelmeyer \\ Universidade de Caxias do Sul, Brasil. \\ http://lattes.cnpq.br/2397483078480061 \\ uilicara@gmail.com \\ Tânia Craco \\ Instituto Federal de Administração RS, Brasil. \\ http://lattes.cnpq.br/0921686278600926 \\ tcraco@yahoo.com.br \\ Flávia Gubert \\ Universidade de Caxias do Sul, Brasil. \\ http://lattes.cnpq.br/5273488527810576 \\ flavia.gubert1@yahoo.com.br
}

\section{Referencing this:}

WELTER, A. S.; REMUSSI, R.; ÓLEA, P. M.; FERNANDES, A. M.; BIEGELMEYER, U. H.; CRCO, T.; GUBERT, F.. Análise de sustentabilidade: Índice De Sustentabilidade Empresarial Bovespa e carteiras 2013, 2014 e 2015. Revista Ibero-Americana de Ciências Ambientais, v.8, n.2, p.307-315, 2017. DOI: http://doi.org/10.6008/SPC2179-6858.2017.002.0025 


\section{INTRODUÇÃO}

No ano de 1987, após a Assembleia Geral da ONU, a presidente da Comissão sobre o Meio Ambiente e Desenvolvimento, apresentou o documento 'Nosso Futuro Comum' um relatório que ficou conhecido como Relatório de Brundtland, em que definiu-se desenvolvimento sustentável como sendo aquele capaz de atender as necessidades das atuais gerações sem comprometer a capacidade das futuras gerações de também o fazê-lo (NEVES, 2011). Deste modo, a sustentabilidade impacta no modo como a sociedade projeta seu desenvolvimento (CAPRA, 2001).

Assim, partindo do pressuposto de que sustentabilidade se trata de um conceito móvel dotado de objetivo permanente, possui parâmetros pela própria sociedade (TACHIZAWA et al., 2008). Deste modo, o tema empresas sustentáveis é amplo e abrangente, configurando-se como um debate relacionado ao fortalecimento de instituições e sistemas de governança que as apoiam. As organizações precisam assegurar que suas atividades principais agreguem valores e sejam conduzidas de forma eficaz. Neste contexto, quando as empresas cumprem requisitos ambientais, recebem a aprovação de empresas sustentáveis, através de indicadores de sustentabilidade em bolsas de valores, como Dow Jones Sustainability Índex de Nova lorque, FTSE 4 GOOD de Londres, Financiall Times Reino Unido e ISE no Brasil, Índice de Sustentabilidade Empresarial da BM\&FBOVESPA, por exemplo.

Com vistas a isso, a investigação realizada teve por objetivo identificar a evolução do número de empresas elencadas no Índice de Sustentabilidade Empresarial (ISE) definido pelo BM\&BOVESPA. Deste modo, além desta introdução, este estudo é composto por mais quatro sessões. O referencial teórico que fornece subsídios para a investigação empírica realizada. A metodologia, que expõem o delineamento da pesquisa, bem como procedimentos de coleta e análise dos dados. Em seguida, a análise e discussão dos resultados, contrastando-os com a literatura e, por fim, as considerações finais, que retomam o objetivo do estudo, apresentam as limitações da pesquisa e sugestões para estudos futuros.

\section{REFERENCIAL TEÓRICO}

\section{Sustentabilidade}

O termo sustentabilidade teve sua origem da conscientização crescente, durante a década de 1980, na qual os países precisavam descobrir maneiras de promover o crescimento de suas economias sem destruir o meio ambiente ou sacrificar o bem-estar das futuras gerações (SAVITZ, 2007). Sustentabilidade se caracteriza como um conceito interdisciplinar, visto que é empregado em distintas áreas do conhecimento e assim pode ser compreendida como uma questão de se estabelecer distribuição igualitária de bem-estar associada aos recursos naturais nas dimensões inter e intratemporal (MACHADO et al., 2009).

Em um estudo considerado clássico sobre o tema, Sachs (1993) identificou cinco dimensões da sustentabilidade, quais sejam: (i) sustentabilidade social: construir uma civilização em que exista maior equidade na distribuição de renda e de bens, que reduza a distância entre os padrões de vida dos ricos e dos pobres; (ii) sustentabilidade econômica: é possível pela alocação e gestão mais eficientes dos recursos e por 
um fluxo constante de investimentos públicos e privados; (iii) sustentabilidade espacial: está voltada para uma configuração rural-urbana mais equilibrada e uma melhor distribuição territorial dos assentamentos humanos e das atividades econômicas, reduzindo a concentração excessiva nas áreas metropolitanas; freando a destruição de ecossistemas frágeis pelo processo de colonização e pelo estabelecimento de uma rede de reservas naturais para proteger a biodiversidade; (iv) sustentabilidade ecológica: intensifica o uso de recursos para propósitos sociais; limitando e substituindo o consumo de recursos naturais não renováveis ou danosos ao meio ambiente; reduzindo o volume de resíduos e de poluição, por meio da conservação de energia e da reciclagem; promovendo a redução do consumo pelos mais ricos; intensificando o desenvolvimento e uso de tecnologias limpas; promovendo o uso de instrumentos econômicos, legais e administrativos por meio de regras adequadas à proteção ambiental. Por sua vez, e; (v) sustentabilidade cultural: privilegia processos de mudança cultural de uma comunidade, que resultem em uma pluralidade de soluções particulares e que respeitem as especificidades de cada ecossistema, cultura e local.

Um dos estudos pioneiros sobre o tema sustentabilidade encontra-se no relatório elaborado pela Comissão Brundtland (ONU, 1991), enfatiza que em toda definição de sustentabilidade está implícita a noção de igualdade social entre diferentes gerações, e em cada geração. As necessidades básicas de grande número de pessoas não estão sendo atendidas, principalmente nos países em desenvolvimento. $O$ aumento da produção deve assegurar aos indivíduos as mesmas oportunidades de realizar suas aspirações de uma melhor qualidade de vida, por meio da redução dos níveis de consumo dos países desenvolvidos e atendimento aos padrões mínimos de consumo nos países em desenvolvimento.

E por fim, o desenvolvimento sustentável deve ser desenvolvido, trabalhado e envolvido de forma global para reverter o quadro de degradação social e ambiental em que se encontra o planeta. Newport et al. (2003) afirmam ainda que as empresas têm um longo caminho a percorrer, assim como há um vasto campo de pesquisa na área da gestão da sustentabilidade.

\section{Sustentabilidade Empresarial}

O tema é bastante amplo, o conceito de empresa sustentável está relacionado com a noção de desenvolvimento sustentável através do compromisso com a sociedade, baseado na ética e promoção dos direitos humanos. Novas teorias surgiram como a Teoria do Stakeholder, onde parte interessada, são pessoas que fazem parte do relacionamento da empresa, porém não fazem parte necessariamente do quadro funcional. A definição de Stakeholder mais criada por Freeman (1984), que o define como "qualquer grupo ou indivíduo que afeta ou é afetado pelo alcance dos objetivos da empresa".

Desenvolveu-se a noção de Responsabilidade Ambiental e a busca por atender a legislação e regulamentações, além da redução de custos através da ecoeficiência. Em 1994, o inglês John Elkington, consolida o termo Triple Bottom Line, que define a responsabilidade por parte das empresas em equilibrar os retornos econômicos, sociais e ambientais em suas operações.

Essa massa consumidora, cada vez mais, representa uma pressão constante sobre as empresas e suas práticas de produção e de prestação de serviços. Isso é muito positivo, pois cria nas empresas a necessidade 
de adaptarem seus procedimentos ou de mudarem sua forma de agir de forma drástica e rápida; sob pena de verem suas vendas (e seus lucros) caírem vertiginosamente de forma perigosa e arriscada. Esse "novo comportamento" acabou recebendo o nome de sustentabilidade empresarial. Desta forma, as empresas acabaram definindo um conjunto de práticas que procuravam demonstrar o seu respeito e a sua preocupação com as condições do ambiente e da sociedade em que estão inseridas ou onde atuam (ABREU, 2008).

\section{Breve Histórico do Índice Empresarial no Brasil}

A educação ambiental, refere-se a um tema relativamente novo, porém a história do meio ambiente e da educação ambiental se difundem e se aperfeiçoam de forma evolutiva. Entre os anos 50 e 70, houve a exclusão de alguns setores, que antes ocorriam apenas por motivos religiosos, porém passam a se alicerçar por motivos morais. Investidores americanos e europeus deixam de alocar recursos em empresas que possuem relação com o regime de apartheid da África do Sul. Porém nessa época ainda havia pouco entendimento sobre seus impactos socioambientais.

Nos anos 80, houve a exclusão de alguns setores relacionados aos armamentos, bebidas alcoólicas e tabaco, conferindo certa evolução para as boas práticas ambientais, sociais e de governança em todos os setores. As empresas envolvidas passaram a procurar uma maneira de desenvolver certa capacidade em atender novas obrigações e licenças legais de operações. Em 1999 foi lançado o Dow Jones Sustainability Indexes, através da Bolsa de Nova lorque, ela apresentou o primeiro índice global dedicado ao mapeamento e acompanhamento do desempenho financeiro das empresas que incorporaram o tema sustentabilidade em seus negócios. Tais empresas começaram a identificar benefícios as práticas de prevenção, poluição e eco eficiência que passou a gerar lucros econômicos.

\section{Índices de Sustentabilidade Empresarial - ISE no Brasil}

As empresas que cumprem requisitos ambientais recebem a aprovação de empresas sustentáveis, através de índices de sustentabilidade em bolsas de valores. Tais indicadores econômicos representam alguns conceitos de análise de balanço empresarial. A partir do balanço patrimonial e da demonstração de resultados, são efetuados cálculos matemáticos, verificando números e propiciando suporte ao processo de entendimento e posicionamento da empresa no mercado financeiro, através de aspectos patrimoniais, tais informações irão agregar suportes sobre os principais dados de rentabilidade da empresa, além de demonstrar sua evolução.

Os documentos comprobatórios de suas boas práticas e participar de um processo competitivo de análises. As empresas que participam do processo de avaliação tornam-se referências em práticas de gestão sustentável no Brasil e no mundo, tal índice atua como instrumento e fator de preparação, atuação e aprimoramento, além do uso de ferramentas de avaliação das práticas de sustentabilidade adotada nas melhores empresas do mundo. E os indicadores não podem serem vistos como estáticos, mas como integradores no decorres do tempo, com o intuito de demonstrar tendências (GALLOPÍN, 1997) 
Para a Bovespa "ISE" é um índice que mede o retorno médio de uma carteira teórica de ações de empresas de capital aberto e listadas na BM\&FBOVESPA com as melhores práticas em sustentabilidade. A participação do meio empresarial junto ao ISE é voluntária, sendo que as empresas devem ser classificadas entre as 200 ações mais líquidas da BM\&FBOVESPA, destas compostas por até 40 empresas. A metodologia de escolha da empresa candidata ao índice é baseada através de um questionário que apresenta questões relacionadas a sustentabilidade e meio ambiente, com sete dimensões determinadas pela Bovespa: (i) natureza do produto: impactos sociais, impactos difusos, princípio da precaução e informação ao consumidor; (ii) mudanças climáticas: política, gestão, desempenho e reporting; (iii) geral: compromissos, alinhamento, transparência, corrupção; (iv) ambiental: política, gestão, desempenho e cumprimento legal; (v) governança corporativa: propriedade, conselho de administração, auditoria e fiscalização, conduta e conflito de interesses; (vi) social: política, gestão, desempenho e cumprimento legal; e (vii) econômica financeira: política, gestão, desempenho e cumprimento legal.

O ISE possui um conselho deliberativo composto por onze instituições representantes, são elas: BM\&FBOVESPA A Nova Bolsa, IBGC Instituto Brasileiro de Governança Corporativa, MMA Ministério do Meio Ambiente, IFC International Finance Corporation, ANBIMA, APIMEC, ABRAPP, Instituto ETHOS, PNUMA, IBRACON Instituto dos Auditores Independentes do Brasil e GIFE. Com a missão de garantir a transparência no processo de construção do índice de seleção das empresas.

No que corresponde ao valor do ISE, estudos da BM\&FBOVESPA, indica ganhos em valores tangíveis e intangíveis, para as empresas que fazem parte da carteira do ISE, os mesmos são definidos da seguinte forma: (i) bom desempenho socioambiental influencia positivamente o desempenho financeiro (retorno sobre o patrimônio e retorno sobre ativos) e vice-versa; (ii) empresas da carteira do ISE apresentam valor de mercado de $10 \%$ a $19 \%$ maior do que o grupo de controle correspondente; (iii) nos dias próximos ao anúncio da carteira do ISE, as ações das empresas participantes apresentam retorno acumulado anormal de 1,9\% em relação às empresas do menor setor; (iv) first mover: estímulo à inovação e compensação dos custos de 'compliance'; (v) ambiente regulatório: vantagem competitiva de adequação prévia à regulamentação; (vi) reputação: diálogo e transparência proativa por parte das empresas; e (vii) conhecimento: iniciativas voluntárias envolvem compartilhamento de conhecimento.

A BOVESPA destaca ainda alguns objetivos estratégicos direcionados ao período de 2011 até 2015, como: (i) ampliação e abertura de informações ao mercado; (ii) aumento e a participação das empresas no processo de seleção; (iii) aumento do volume de recursos investidos e produtos atrelados ao ISE a fim de torná-los um benchmark de investimentos; (iv) fortalecimento dos canais de comunicação e de diálogo com as partes de interesse; (v) aperfeiçoamento do escopo e processos de elaboração do questionário; (vi) refinamento e aperfeiçoamento da metodologia; e (vii) verificação dos processos de seleção das empresas.

É certo afirmar que os índices são instrumentos que norteiam e tornam visíveis as variáveis de determinados processos, facilitando a tomada de decisões e permitindo o desenvolvimento e acompanhamento de atividades relacionadas aos diversos campos da sustentabilidade, favorecendo a compreensão e direcionando os envolvidos na sistematização das informações. 


\section{METODOLOGIA}

A pesquisa desenvolvida teve abordagem quantitativa, e no que se refere a finalidade, consistiu em pesquisa exploratória e descritiva, adotando como procedimentos técnicos pesquisa documental, cujos dados foram obtidos por meio de consulta na carteira do Índice de Sustentabilidade Empresarial (ISE) divulgado pela BOVESPA. Assim, a análise dos dados ocorreu através de comparação entre as três últimas carteiras ISE/BOVESPA, correspondentes aos anos 2013, 2014 e 2015 respectivamente.

\section{RESULTADOS E DISCUSSÃO}

\section{Carteira do índice de sustentabilidade empresarial - ISE 2015 - 10a Carteira}

O período de vigor da carteira corresponde entre os dias 05 de janeiro de 2015 até 02 de janeiro de 2016, conforme demonstra a Quadro 1. No que corresponde aos dados da BM\&FBOVESPA, o raio x da décima carteira ISE mostra os seguintes dados:

$65 \%$ das empresas participantes dessa carteira possuem comitê de sustentabilidade que se reporta ao conselho de administração, sendo que em 2013 esse percentual correspondeu a $58 \%$;

98\% das empresas contam com diretoria responsável pelas questões referente à sustentabilidade que se reporta diretamente ao primeiro escalão, sendo que no ano de 2013 esse percentual correspondeu a 95\%;

$83 \%$ das empresas contam com políticas corporativas relacionadas as mudanças de clima aprovadas pelo CA ou pela direção;

$65 \%$ das empresas possuem um sistema de remuneração variável com previsão e correlação entre riscos, remuneração destinada ao quadro funcional, e o resultado da empresa, sendo que em 2013, esse percentual correspondeu a 53\%;

$84 \%$ das empresas eleitas ao ISE desta carteira, declaram a divulgação de inventário de emissões de GEE, destas $67 \%$ comprometem-se com metas de redução, $49 \%$ atingiram os seus objetivos ou ultrapassaram as metas e $33 \%$ destas companhias não estabeleceram suas metas;

$77 \%$ das empresas que compõem a carteira, praticam a incorporação de requisitos ambientais, em projetos de novos produtos e serviços.

Quadro 1: Carteira ISE/Bovespa 2015.

\begin{tabular}{|c|c|c|c|c|}
\hline AES TIETE & CEMIG* & ELETROBRAS* & ITAUSA* & SABESP \\
\hline B2WDIGITAL* & CIELO* & ELETROPAULO* & ITAU-UNIBANCO* & SANTANDER BR* \\
\hline BANCO DO BRASIL* & COELCE* & EMBRAER & JSL* & SUL AMERICA* \\
\hline BICBANCO* & COPEL* & EVEN* & KLABIN S/A* & TELEFÔNICA BRASIL \\
\hline BRADESCO* & CPFL ENERGIA* & FIBRIA* & LIGHT S/A* & TIM PART S/A \\
\hline BRASKEM* & DURATEX* & FLEURY* & LOJAS AMERICANAS* & TRACTEBEL* \\
\hline BRF SA* & ECORODOVIAS* & GERDAU & LOJAS RENNER* & VALE* \\
\hline CCRSA & ENERGIAS BR(EDP)* & GERDAU MET & NATURA* & WEG* \\
\hline
\end{tabular}

Através de divulgação no site da BM\&FBOVESPA, constatou-se que das quarenta empresas selecionadas para a 10a carteira ISE, quatro delas são novas: JSL, B2W Digital, Lojas Americanas e Lojas Renner, destas as três últimas são responsáveis pela inserção do setor de comércio na carteira. O número de empresas que autorizaram a abertura do questionário elevou-se de 22 para 34 , do total da 10a carteira, demonstrando mais transparência por parte de tais empresas. 


\section{Carteira do índice de sustentabilidade empresarial - ISE 2014 - 9a Carteira}

O período de vigor da carteira correspondeu aos dias 06 de janeiro de 2014 até 02 de janeiro de 2015, conforme demonstra Quadro 2. No que corresponde aos dados da BM\&FBOVESPA, o raio $x$ da nona carteira ISE mostra os seguintes dados:

$100 \%$ das empresas participantes dessa carteira publicaram o relatório de sustentabilidade, sendo que destes $95 \%$ no modelo GRI, em 93\% das empresas houve envolvimento direto dos administradores na elaboração deste;

$100 \%$ das companhias comprovaram o compromisso relacionado ao desenvolvimento sustentável formalmente inserido na estratégia da empresa;

95\% das companhias demonstraram possuir diretoria que se reporta ao primeiro escalão;

$58 \%$ das companhias demonstraram possuir comitê de sustentabilidade que se reportou ao conselho de administração.

Quadro 2: Carteira ISE/ BOVESPA.

\begin{tabular}{|l|l|l|l|l|}
\hline AES TIETË & CESP & EDP* & ITAÚ-UNIBANCO* & SANTANDER \\
\hline BANCO DO BRASIL* & CIELO* & ELETROBRAS* & ITAUSA* & SUL AMÉRICA* \\
\hline BIC BANCO* & COELCE* & ELETROPAULO* & KLABIN* & SUZANO \\
\hline BRADESO & COPASA* & EMBRAER & LIGHT* & TELEFÔNICA \\
\hline BRASKEM & COPEL* & EVEN* & MET GERDAU & TIM \\
\hline BRF & CPFL & FIBRIA & NATURA* & TRACTEBEL \\
\hline CCR & DURATEX* & FLEURY & OI & VALE* \\
\hline CEMIG* & ECORODOVIAS & GERDAU & SABESP & WEG* \\
\hline
\end{tabular}

Através de divulgação no site da BM\&FBOVESPA, constatou-se que das trinta e sete empresas selecionadas na carteira anterior também foram selecionadas para a 9a carteira ISE, quatro delas são novas: Cielo, Embraer, Fleury e Klabin. São responsáveis pela inserção de três novos setores: Serviços Financeiros Diversos, Material de Transporte e Serviços Médicos Hospitalares, Análises e Diagnósticos.

O número de empresas que autorizaram a abertura do questionário elevou-se de 14 para 22 na 9a carteira ISE, demonstrando o aumento de empresas interessadas em demonstrar transparência em suas atividades.

\section{Carteira do índice de sustentabilidade empresarial - ISE 2013 - 8a Carteira}

O período de vigor da carteira correspondeu aos dias 07 de janeiro de 2013 até 03 de janeiro de 2014, conforme Quadro 3. No que corresponde aos dados da BM\&FBovespa, o raio x da oitava carteira ISE mostra os seguintes dados:

$100 \%$ das empresas possuem compromisso formalmente inserido em suas estratégias empresariais com relação ao desenvolvimento sustentável;

$100 \%$ das empresas possuem a publicação deste compromisso em sua web site, sedo de livre acesso;

97\% das empresas possuem programa de educação e sensibilização sobre o tema proposto;

92\% das companhias inseriram-se publicamente e formalmente em compromissos voluntários de desenvolvimento sustentável, comprometendo-se com as unidades subsidiárias ou controladas;

100\% das empresas pertencentes ao ISE 2013, publicaram relatório de sustentabilidade; 
Destas, 92\% guiaram-se através das diretrizes da GRI, para efetuar a elaboração deles;

Para definição do relatório de sustentabilidade em $86 \%$ dos casos, houve o envolvimento direto dos administradores da empresa para tal.

Quadro 3: 8a Carteira ISE/ BOVESPA.

\begin{tabular}{|l|l|l|l|l|}
\hline AES TIETÉ & COPASA & EVEN & LIGHT* $^{*}$ & TIM \\
\hline BANCO DO BRASIL** & COPEL & ELETROBRAS* & NATURA** & TRACTEBEL \\
\hline BIC BANCO** & PPFL & ECORODOVIAS & OI & ULTRAPAR \\
\hline BRADESCO & COELCE** & FIBRIA & SABESP & VALE* \\
\hline BRASKEM & CCR** & GERDAU & SANTANDER & WEG* \\
\hline BRF & ELETROPAULO** & GERDAU MET & SUZANO & \\
\hline CEMIG* & ECORODOVIAS & ITAÚ UNIBANCO & SUL AMÉRICA* & \\
\hline CESP & EDP** & ITAÚSA & TELEFÔNICA & \\
\hline
\end{tabular}

*Empresas que autorizaram a publicação de suas respostas em 2012. **Empresas que autorizaram a publicação de suas respostas em 2012 e 2011.

Através de divulgação no site da BM\&FBOVESPA, constatou-se que das trinta e sete empresas selecionadas na carteira anterior, 35 também foram selecionadas para a 8a carteira ISE, duas delas são novas: Telefônica e WEG. A WEG é responsável por trazer ao ISE o setor de Máquinas e Equipamentos, Motores e Compressores.

O número de empresas que autorizaram a abertura do questionário elevou-se de 8 para 14 na 8 a carteira ISE, demonstrando o aumento de empresas interessadas em demonstrar transparência em suas atividades.

\section{CONSIDERAÇÕES FINAIS}

As organizações vivem um momento de constantes transformações e competitividade aliadas as novas tecnologias e demandas de sustentabilidade. Assim, para atender as necessidades do mercado é necessário atenção as necessidades alheias. Deste modo, a pesquisa realizada teve por objetivo analisar a evolução de tais necessidades, bem como, demonstrar a evolução da sustentabilidade por parte das empresas norteadoras e reguladoras.

Também foram definidas as ferramentas necessárias para a maximização do índice de sustentabilidade das empresas. Por meio da pesquisa efetuada, foi possível perceber o interesse por parte do meio empresarial em sustentabilidade, através da evolução constante e do crescimento dos setores envolvidos no ISE. Deste modo, a pesquisa desenvolvida permitiu comparar as três últimas carteiras do ISE/BOVESPA, o que demonstrou a maximização do número de empresas que se interessam pelas questões ambientais com o passar dos anos.

Todavia, reconhecem-se as limitações do estudo quanto ao aprofundamento das descrições acerca das ferramentas ambientais. Para estudos futuros, recomenda-se a aplicação de uma pesquisa quantitativa por meio de uma survey nas empresas elencadas nas carteiras do ISE, a fim de identificar as variáveis que interferem nas questões ambientais dessas empresas. Também se sugere a aplicação de uma pesquisa qualitativa através de estudo de casos a fim de analisar os aspectos motivadores e moderadores dos aspectos ambientais. 


\section{REFERÊNCIAS}

ABREU, C.. Você Sabe o que é Sustentabilidade Empresarial. 2016.

CAPRA, F.. Ponto de Mutação. São Paulo: Cultrix, 2001.

ONU. Comissão Mundial Sobre Meio Ambiente e Desenvolvimento - CMMAD. Nosso futuro comum. 2 ed. Rio de Janeiro: FGV, 1991.

FREEMAN, R. E.. Strategic management: a stakeholder approach. Boston: Pitman, 1984.

GALLOPÍN, G. C.. Indicators and their use: information for decision making. In: MOLDAN, B.; BILHARZ, S.; MATRAVERS, R.. Sustainability indicators: a reporto $n$ the Project on indicators of sustainable development. Chichester: Wiley and Sons, 1997.

MACHADO, M. R.; MACHADO, M. A. V.; CORRAR, L. J.. Desempenho do índice de sustentabilidade empresarial (ISE) da Bolsa de Valores de São Paulo. Revista Universo Contábil, v.5, n.2, p.24-38, 2009.
NEVES, L. S.. Sustentabilidade Anais de textos selecionados do V Seminário Sobre Sustentabilidade. Rio de Janeiro: Juruá, 2011.

NEWPORT, D.; CHESNES, T.; LINDNER, A.. The "environmental sustainability" problem: ensuring that sustainability stands on three legs. International Journal of Sustainability in Higher Education, Bradford, v.4, n.4, p.357363, 2003.

SACHS, I.. Estratégias de Transição para do século XX: Desenvolvimento e Meio Ambiente. São Paulo: Studio Nobel, 1993.

SAVITZ, A.. A Empresa Sustentável: O verdadeiro sucesso é o lucro com responsabilidade social e ambiental. Rio de Janeiro: Elsevier, 2007.

TACHIZAWA, T.. Gestão ambiental e responsabilidade social corporativa. São Paulo: Atlas, 2002. 